\title{
Principales factores de éxito en el ámbito de la administración pública
}

\author{
Key success factors in the public administration field
}

\section{RESUMEN:}

Este trabajo se basa en la metodología de sistematización de experiencias a partir de un enfoque cualitativo, cuyo fin es contribuir a la generación de conocimiento para el análisis y discusión de una cuestión fundamental: la gestión pública.

La propuesta parte del intercambio académico a nivel de organizaciones de América Latina, en el cual se comparten las vivencias que permiten enriquecer la discusión teórica y, con mirada crítica, abordar su aplicabilidad en la cotidianeidad laboral. Se describe los principales factores de éxito para lograr un mejor desempeño dentro del ámbito de la administración pública. La gestión institucional se ve fortalecida por medio de una reflexión constante con el quehacer individual que aporta a su vez valor agregado al bienestar colectivo. Se aporta una visión general que abarca el conocimiento de los elementos del contex to internacional y nacional, analizando las buenas prácticas de la nueva gestión pública.

\section{ABSTRACT:}

This essay is based on the methodology of systematization of experiences as part of a qualitative approach, whose aim is to contribute to the generation of knowledge for the analysis and discussion of a fundamental issue: public management.

The proposal is based on the academic exchange at the level of Latin American organizations, in which the experiences that allow enriching the theoretical discussion and, with a critical perspective, addressing its applicability in the daily work are shared. This article describes the key success factors to achieve a better performance within the scope of public administration. Institutional management is strengthened by means of a constant reflection on the individual endeavor that provides in turn added value to the collective welfare. There is an encompassing overview of the knowledge of the international and national context elements by analyzing the good practices of the new public management.

\begin{tabular}{l|l}
\multicolumn{1}{c|}{ PALABRAS CLAVES } & \multicolumn{1}{|c}{ KEYWORDS } \\
ÍNDICE DE COMPETITIVIDAD & GLOBAL COMPEIITIVENESS \\
GLOBAL, ÍNDICE DE PROGRESO & INDEX, SOCIAL PROGRESS \\
SOCIAL, MODELO DE CAMBIO & INDEX, INTENTIONAL \\
INTENCIONADO, MODELO DE & CHANGE MODEL, DECISION \\
TOMA DE DECISIONES, VALOR & TAKING MODEL, PUBLIC \\
PÚBLICO & VALUE
\end{tabular}

\section{RESUMO:}

Este trabalho baseia-se na metodologia de sistematização de experiências a partir de um enfoque qualitativo, cujo objetivo é contribuir para a geração de conhecimento para a análise e discussão de uma questão fundamental: a gestão pública.

A proposta nasce do intercâmbio acadêmico ao nível das organizações da América Latina, no qual são compartilhadas as experiências que permitem o enriquecimento da discussão teórica e, com uma visão crítica, abordar sua aplicabilidade no trabalho cotidiano. São descritos os principais fatores de sucesso para obter melhor desempenho no âmbito da administração pública. A gestão institucional fica fortalecida por meio da reflexão constante com o trabalho individual que, por sua vez, agrega valor ao bem-estar coletivo. É fornecida uma visão geral que abrange o conhecimento dos elementos do contexto internacional e nacional, analisando as boas práticas da nova gestão pública.

\section{RÉSUMÉ:}

Cet essai est basé sur la méthodologie de systématisation d'expériences à partir d'une approche qualitative, dont le but est de contribuer à la production de connaissances pour l'analyse et la discussion d'une question fondamentale: l'administration publique. La proposition est basée sur l'échange académique au niveau des organisations d'Amérique Latine, au moyen duquel s'effectue un partage des expériences qui permettent d'enrichir la discussion théorique et, avec un regard critique, d'aborder sa mise en application dans le travail quotidien. Il y a une description des principaux facteurs de succès pour atteindre une meilleure performance dans le cadre de l'administration publique. L'administration institutionnelle est consolidée par le biais d'une réflexion constante sur le travail individuel qui donne à la fois une valeur ajoutée au bien-être collectif. Il s'agit d'une vision générale qui couvre l'ensemble des éléments du contexte international et national, par l'analyse des bonnes pratiques de la nouvelle administration publique.

PALAVRAS CHAVES
ÍNDICE DE COMPETITIIIDADE
GLOBAL, INDICE DE
PROGRESSO SOCIAL, MODELO
DE MUDANÇA INTENCIONAL,
MODELO DE TOMADA DE
DECISAOO, VALOR PÚBLICO

\section{MOTS CLÉS}

INDICE DE COMPETITIVITE GLOBALE, INDICE DE PROGRES SOCIAL, MODELE DE CHANGEMENT INTENTIONNEL, MODELE DE PRISE DE DECISIONS, VALEUR PUBLIQUE 


\section{INTRODUCCIÓN}

La sistematización de experiencias se ha convertido en una herramienta de conocimiento y aprendizaje en diversas esferas de acción. Este artículo pretende contribuir al análisis y discusión de los principales elementos considerados como factores de éxito en la administración pública.

El referente conceptual lo constituye la experiencia académica del Programa Ejecutivo en Administración Pública del Incae-Universidad de Georgetown. Este curso consistió en un programa de doble titulación dada por la Escuela de Negocios Incae y la Escuela de Negocios McDonough de la Universidad de Georgetown, modalidad de aprendizaje b-learning o híbrida, al combinar capacitación presencial y educación en línea. Como objetivos, consideró los siguientes: a) aprender a mejorar el marco de toma de decisiones, b) diseñar e implementar sistemas de medición del rendimiento, c) aprender a establecer y medir objetivos, d) definir, ejecutar y evaluar proyectos públicos y e) motivar personas y dinamizar equipos. Se estructuró en tres módulos, con lecturas previas a las sesiones presenciales y con base en preguntas clave de discusión. El primer módulo fue realizado en Ciudad de Panamá, del 21 al 25 de agosto; el segundo en Alajuela, Costa Rica, del 25 al 29 de septiembre y el último en Washington D.C., Estados Unidos, del 23 al 25 de octubre de 2017. Como parte de su metodología, el curso utilizó plataforma virtual, análisis de debate en grupos, conferencias magistrales, simulaciones, herramientas de desarrollo de liderazgo de equipos y organizacional, y visitas a organizaciones, como parte del proceso de aprendizaje.

Con respecto a las calidades del profesorado, se destacó por contar con un alto nivel académico de sus profesores, la mayoría de ellos con nivel de doctorado o aspirantes a ello, en universidades de alto prestigio internacional, tales como la Universidad de Harvard, la Universidad de Pensilvania y la Universidad de Oxford. Aunado a ello, la trayectoria profesional resaltó la experiencia en organizaciones gubernamentales y sector privado, en puestos de alta exigencia y responsabilidad.

El grupo de estudiantes fue intrarregional, con personas provenientes de Panamá, Honduras, Costa Rica, Venezuela, Perú y Paraguay, con experiencia en puestos del sector público, permitiendo con ello enriquecer la vivencia del proceso de aprendizaje.

Cabe mencionar que, según los Lineamientos de Política Institucional 2015-2019 de la UNED, en su capítulo 4 "La Gestión Universitaria", lineamiento 141, se indica: "Debe facilitarse la asistencia a actividades académicas, tales como congresos y conferencias, además de promoverse todo tipo de intercambio académico". La premisa de esta propuesta es que el individuo debe autorrealizarse para aportar al objetivo común, dado que las labores se convierten en una parte integral de su proyecto de vida, esto evidenciado en los lineamientos 134 y 135 , que instan a generar una cultura de incentivos en el personal y formación continua.

De igual forma, lo expuesto en este trabajo guarda relación con el lineamiento 107, que a la letra dice:

El modelo de gestión de la universidad debe elaborarse específicamente en función de la academia y con el fin de fortalecer su calidad: debe fundamentarse en enfoques y conceptos apropiados, pertinentes y actualizados de las ciencias sociales y la administración, en perspectivas científicas y filosóficas pertinentes para comprender las organizaciones humanas y en las concepciones éticas y filosóficas fundamentales de la universidad y de su papel en nuestra sociedad, según ello ha quedado formulado en los documentos fundadores de la UNED, los acuerdos de los congresos universitarios y en estos lineamientos."

En el marco de estos lineamientos, la UNED se suscribe en el año 2010 al proyecto del Acuerdo de Mejoramiento Institucional (AMI), empréstito internacional con el Banco Mundial, que inició su implementación en junio del 2013 a concluir en diciembre de 2019. La naturaleza del proyecto con financiamiento internacional trae consigo una serie de elementos clave, como, por ejemplo, apoyo político, objetivos de desarrollo y planificación clara de la ruta de trabajo, 
la creación de sinergia organizacional y la posibilidad de desarrollo de equipos de trabajo, una ágil toma de decisiones y una lectura del entorno en el que se desarrolla, así como una rendición de cuentas y evaluación constante, por lo que la experiencia académica realizada y sistematizada en este artículo ha sido, sin duda, una contribución significativa para la aplicabilidad de la teoría a la práctica en dicho proyecto.

A partir de lo anterior, este trabajo propone contribuir a la discusión de la gestión institucional y sus principales factores clave de éxito.

\section{DESARROLLO}

En este apartado, se analizan los elementos clave que se deben considerar para mejorar el perfil profesional en el sector público, con el fin de coadyuvar a una gestión eficiente. Se parte del argumento de que, al mejorar las cualidades y capacidades del talento humano, se logra aportar a un mejor desempeño institucional.

La siguiente figura muestra los factores clave de éxito que se analizan como los principales elementos para la comprensión, aprendizaje y aplicabilidad en la gestión.

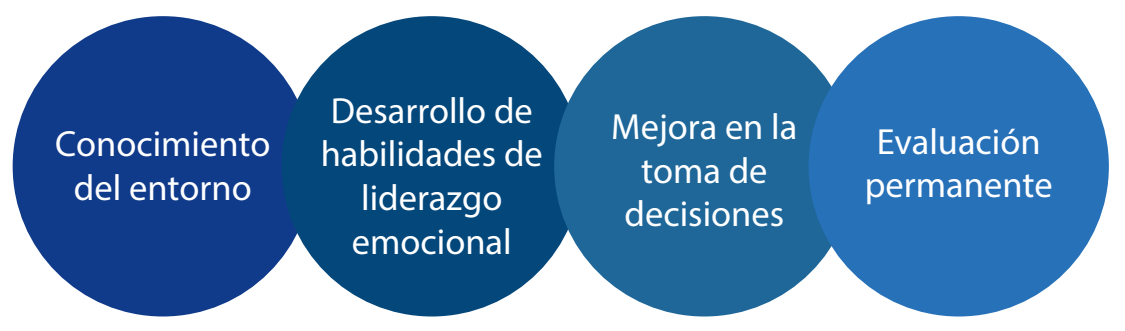

Figura 1. Factores clave de éxito

Fuente: Elaboración propia con base en las fuentes consultadas.

\section{Conocimiento del entorno o contexto}

Uno de los principales factores guarda relación con el conocimiento de lo que sucede en el exterior, es decir, de aquellas variables externas que inciden en la institución. Se menciona, entre ellas, la comprensión del índice de competitividad global y el índice de progreso social, como valor agregado en la función pública. 
A continuación, se ofrece en forma somera una explicación de estos elementos.

\section{Índice de competitividad global}

El Foro Económico Mundial define la competitividad como "el conjunto de instituciones, políticas y factores que determinan el nivel de productividad de un país” (Foro Económico Mundial, 2017, citado por INCAE Business School, pág. 2, 2017). Para dicho ente, la productividad conduce al crecimiento y al nivel de ingresos, lo cual se relaciona con bienestar humano.

Según el Centro Latinoamericano para la Competitividad y el Desarrollo Sostenible (CLACDS, 2017), el índice "captura el conjunto de instituciones, políticas y factores que determinan el crecimiento económico sostenible y ayudan a explicar por qué algunos países son más exitosos que otros en elevar, de manera sostenible, su productividad, niveles de ingreso y oportunidades para sus respectivas poblaciones.” ${ }^{1}$

Este índice se mide con doce pilares, agrupados en tres subíndices, tal como se muestra en la siguiente figura:

\section{Requerimientos básicos}

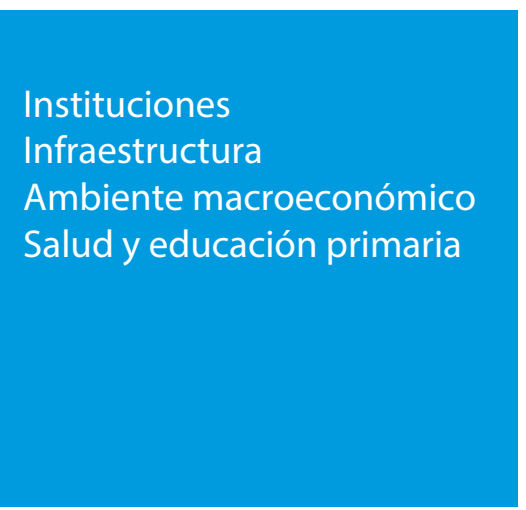

\section{Potenciadores de eficiencia}

Educación superior y capaci

tación

Eficiencia en el mercado de

bienes

Eficiencia en el mercado

laboral

Desarrollo de mercados

financieros

Preparación tecnológica

Tamaño del mercado
Factores de innovación y sofisticación

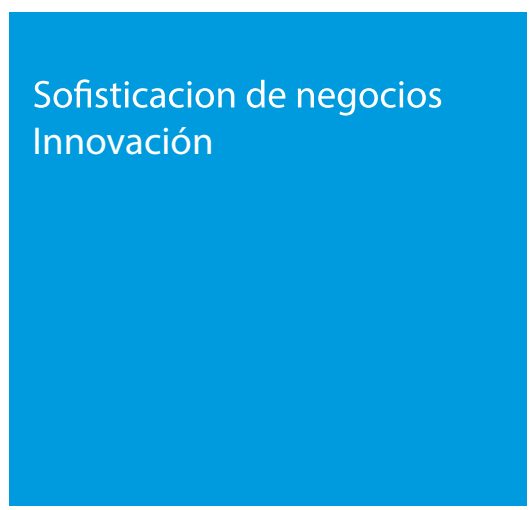

Figura 2. Subíndices y pilares de índice de competitividad global

Fuente: Elaboración propia a partir de Foro Económico Mundial (2017). 
Según el Foro Económico Mundial, se entiende el pilar de instituciones como elmarco legal y administrativo en el que los individuos, las empresas y los Gobiernos interactúan para generar riqueza.. La infraestructura está relacionada con carreteras, puertos, transporte aéreo y otros, donde la clave es que sea extensa y eficiente para que la economía funcione de forma efectiva. Por su parte, el ambiente macroeconómico es importante, porque la economía de un país no puede crecer de forma sostenida si este no es estable. La salud y educación primaria son relevantes debido a que la inversión en servicios de salud es fundamental para tener una fuerza laboral saludable y así evitar disminuciones en la productividad y competitividad.

Como lo indica el Observatorio de Desarrollo de la Universidad de Costa Rica, la competitividad toma en consideración la cantidad y la calidad de la educación básica, debido a su estrecha relación con la eficiencia y la capacidad de innovación de cada trabajador.

Con respecto a educación superior y capacitación, se considera la educación secundaria, superior y técnica, así como la calidad de la educación general, al ser elementos que permitan que las economías trasladen sus actividades de mayor valor agregado.

Con respecto a la eficiencia en el mercado de bienes, se entiende como la competencia saludable de mercado, doméstica y extranjera es importante para incentivar la eficiencia de mercado y así, la productividad empresarial, de forma que las empresas más eficientes, produciendo bienes demandados en el mercado, sean las que prosperen.

La eficiencia en el mercado laboral se relaciona con la flexibilidad para mover trabajadores de una actividad económica a otra de forma rápida y con bajo costo, y de proveer incentivos para que el talento humano mejore su desempeño y haya un ambiente empresarial que permita la igualdad de género. Con relación al desarrollo de mercados financieros, se entiende que el sector bancario debe ser confiable y transparente para los actores económicos. En lo que concierne a preparación tecnológica, esta mide la agilidad que tiene una economía para adoptar las tecnologías existentes con el fin de mejorar la productividad de sus industrias. Por último, para el subíndice de potenciadores de eficiencia, el tamaño del mercado afecta a la economía de un país, en tanto pueda generar economías de escala.

Por su parte, en relación con los factores de innovación y sofisticación, el Foro Económico Mundial indica que las prácticas empresariales sofisticadas conducen a una mayor eficiencia en la producción de bienes y servicios. Por ello, en el pilar de sofisticación de negocios, considera los conglomerados o clústeres, al generar mayores oportunidades e incrementar la eficiencia. La innovación es de gran valor para las economías, al permitir el conocimiento y la posibilidad de generar valor agregado al adaptarse a las tendencias tecnológicas.

Esbozado el panorama general, cabe destacar que, según el índice de competitividad global para el período 20162017, Costa Rica se ubica en el puesto 54, al bajar dos posiciones con relación a los años 2015-2016. Se destacan en las primeras tres posiciones Suiza, Singapur y Estados Unidos. Si se analiza América Latina, el país que se destaca es Chile, en el puesto 33, y en la región centroamericana se destaca Panamá, en la posición 42.

En el reciente índice de competitividad global para el año 2017-2018, continúa Suiza encabezando la lista; en segundo lugar Estados Unidos y en tercero Singapur. Chile mantiene su puesto 33, Panamá baja al 55 y Costa Rica mejora en la posición 47 .

Nuestro país se destaca como el mejor de América Latina en el pilar de salud y educación primaria, superado solo por Finlandia, que se clasifica como el primero a nivel global. Se resalta también la posición de Costa Rica en sofisticación de negocios e innovación, ocupando el primer lugar en América Latina, superado por Suiza como el mejor país a nivel global en esta materia. Con relación a educación superior y capacitación, Costa Rica, obtiene un puntaje de 5,1 en una escala del 1 al 7, donde el 1 representa la clasificación más baja y el 7 la más favorable. Para el caso de América Latina, Chile obtiene el mejor puntaje, superado a nivel general por Singapur. Según el informe, entre los elementos a mejorar para Costa Rica, se encuentran la infraestructura, en términos de calidad y de carreteras y edificaciones, el equilibrio presupuestario del Gobierno y la burocracia en trámites. 
A partir de lo anterior, se evidencia que la función de la universidad pública es pilar y potenciador de eficiencia para la competitividad de un país, lo cual conlleva a reflexionar para nuestro quehacer que el conocimiento del entorno debe revisarse a la luz de elementos macro que obligan a un análisis continuo de la coyuntura país para propiciar acciones en un marco de responsabilidad.

\section{Índice de progreso social}

El índice de progreso social es "la capacidad de una sociedad de satisfacer las necesidades humanas fundamentales de sus ciudadanos, de establecer cimientos que permitan mejorar y mantener la calidad de vida de sus ciudadanos y comunidades, y de crear las condiciones para que todos los individuos alcancen su pleno potencial” (Porter y Stern, 2017, pág.3).

Los autores señalan que, "este índice se concibe con el objetivo de que sea una herramienta práctica que ayude a los líderes y profesionales del Gobierno, el sector privador y la sociedad civil a implementar políticas y programas que impulsen un progreso social más acelerado" (Porter y Stern, 2017, pág.16).

El marco contempla los siguientes componentes:

\section{Necesidades humanas básicas}

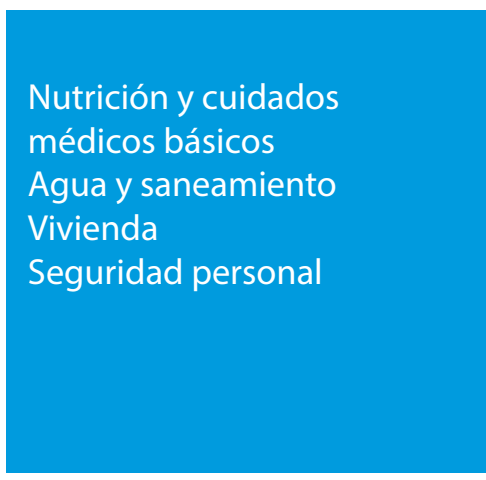

\section{Fundamentos del bienestar}

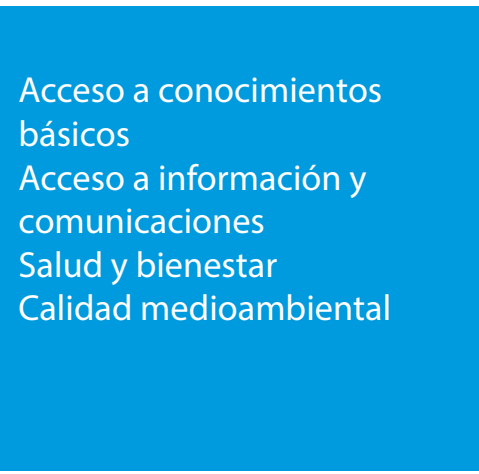

\section{Oportunidades}

Derechos personales

Libertad personal y de

elección

Tolerancia e inclusión

Acceso a Educación Superior

Figura 3. Índice de progreso social

Fuente: Elaboración propia a partir de "Informe Global de Competitividad, 2017" (pág. 12) 
En esta medición se agrupan los países del más alto al más bajo en progreso social, clasificados de "progreso social muy alto" a "progreso social muy bajo”. De 128 países que se incluyen en la medición, Costa Rica está en el puesto 28, con un puntaje de 81,03, ubicada en un nivel de progreso social alto. Según lo anterior, los países ubicados en este rango logran un desempeño en el nivel más alto en nutrición y cuidados médicos básicos, agua y saneamiento, pero rezagados en liberta personal y de elección y en tolerancia e inclusión.

Puede apreciarse el acceso a educación superior como oportunidad para influir en el progreso social. Es indiscutible la contribución del sistema de educación superior, especialmente la UNED, como motor de desarrollo social, llevando acceso, cobertura y equidad a la población. Con esto, se demuestra que la competitividad no solo se basa en producción, sino que contiene un factor de progreso social, en el cual, como universidad, contribuimos con creces. Esto debe ser un estímulo para que elijamos, como personas del sector universitario, un quehacer eficiente como estandarte en nuestro accionar.

Sumado a la comprensión y análisis del índice de competitividad global y al índice de progreso social, es básico el conocimiento del diagnóstico económico nacional, tomando en cuenta, como indicadores primordiales, la producción y el empleo, las cuentas del sector externo (balanza de pagos, reservas monetarias internacionales y deuda externa) y los macroprecios como la inflación, la tasa de interés y el nivel de salarios; esto en procura de comprender el comportamiento de la economía y con ello influir en la toma de decisiones.

Lo expuesto implica un desafío en el quehacer público, dado que nos obliga a trascender más allá del ámbito inmediato, tomando en cuenta que es imprescindible comprender y aprender con mirada crítica y autorreflexiva el accionar dentro de un sistema complejo.

\section{Desarrollo de habilidades de liderazgo emocional}

En este apartado, se hace referencia a un componente fundamental, como lo es el talento humano de una organización. Como indica Jericó (2008, pág.7xddo), el profesional con talento es "un profesional comprometido que pone en práctica sus capacidades para obtener resultados superiores en un entorno y organización determinados”.

Para lograr lo anterior, se hace evidente que es necesario una estructura organizacional que permita invertir en las personas, en generar una motivación constante que lleve a crear equipos de alto desempeño. De la evidencia empírica y del intercambio de opiniones y buenas prácticas, se hace notorio que la universidad tiene camino por recorrer en esta línea. Sin embargo, desde una construcción positiva, se puede iniciar comprendiendo la teoría de los autores sobre liderazgo y aplicar sus enseñanzas de una forma individual que sume a la colectividad.

Al respecto, la teoría del cambio intencionado de Richard Boyatzis indica que el reto de crear y mantener un liderazgo excelente es reconocer y dirigir su propio proceso de aprendizaje. Para Boyatzis y McKee(2003), el modelo puede ayudar a la gente a iniciar una transformación personal, con éxito, emoción y entusiasmo.

El modelo establece cinco fases a seguir:

1. Mi yo ideal: lo que se quiere de la vida y qué dirige su visión personal de futuro,

2. Mi yo real: análisis de puntos fuertes y débiles entre el yo ideal y su actual realidad.

3. Mi agenda de aprendizaje: aprovechar las fortalezas identificadas y trabajar al mismo tiempo en mejorar las debilidades.

4. Experimentar y poner en práctica nuevos hábitos: lo que se aprende, llevarlo a la práctica.

5. Establecer relaciones personales y cercanas: relaciones de confianza que apoyen el proceso.

Este modelo se encauza dentro del liderazgo emocional, habilidad que debe ser cultivada para el manejo profesional individual y colectivo. 
De esta forma, como aspecto innovador, se señala la creación de equipos de alto desempeño para una eficiente gestión pública.

Los equipos de alto desempeño guardan cuatro principios indispensables:

1. Incluir personas con fuerte disposición para ejecutar, la cual permita la movilización del equipo hacia el logro de la meta.

2. Claridad en el objetivo a alcanzar; sin esta condición, puede ocurrir una dispersión de energía y esfuerzo.

3. Claridad en las razones por las que se hace.

4. Entender cómo se hace, para disminuir la curva de aprendizaje.

Lograr equipos de alto desempeño inicia con la identificación preliminar de las características de un equipo disfuncional. La primera de ellas es la ausencia de confianza, al existir miembros del equipo que no se abran a la aceptación de errores y debilidades que fomentan la confianza; esto provoca la segunda disfunción, que es el temor al conflicto, lo cual evita discusiones con un debate abierto. Sin un intercambio y discusión de opiniones, se puede producir en un equipo la falta de compromiso, llevando a evadir responsabilidades, poner los intereses personales por encima de los de la organización y, de esta forma, no prestar atención a los resultados.

En este entramado, es particular el énfasis que debe darse a las personas que forman parte de la organización, estableciendo un modelo de gestión del talento humano que tengan como elemento la innovación para impactar positivamente en el crecimiento institucional. El reto está en mantener de manera intrínseca el espíritu y esfuerzo para generar cambios de actitudes que generen acciones colectivas de alto desempeño. Las opciones de formación del personal son imprescindibles para el logro de lo anterior, tanto por su proceso de aprendizaje como por la internacionalización que conlleva.

\section{Mejora en la toma de decisiones}

La toma de decisiones en toda organización es crucial y, en el ámbito de la administración pública, no es la excepción. No obstante, la complejidad institucional y la falta de información precisa pueden hacer que en muchas ocasiones se den limitaciones o se presenten debilidades en este aspecto.

Por lo tanto, se propone aplicar el modelo de toma decisiones de la Universidad de Harvard, como se indica en las siguientes líneas:

\section{Modelo de toma de decisiones en la administración pública}

El modelo de Harvard para la toma de decisiones incluye cinco pasos:

1. Definir el problema: se relaciona con identificar qué es lo que está pasando, cuáles son los síntomas, identificar problemas y fijar prioridades.

2. Fijar los objetivos y criterios: determinar estándares para evaluar las posibles alternativas de acción.

3. Generar alternativas: implica comenzar con lluvia de ideas, realizar una lista en forma de árbol, desechar las eliminables y combinar las que son mutuamente excluyentes.

4. Evaluar las consecuencias: trata de imaginar qué pasaría con cada alternativa, aplicar criterios y considerar aspectos cualitativos y cuantitativos.

5. Tomar la decisión: seleccionar la alternativa que mejor satisfaga, midiendo la capacidad de ejecución y analizando el nivel de tranquilidad percibida en la toma de decisiones. 
Junto al modelo de toma de decisiones, se visualiza el análisis de problemas decisionales, el cual sigue la siguiente ruta:

- Definición y justificación del problema, para poder tener una comprensión global.

- Análisis del medio ambiente, con el fin de encontrar los puntos fuertes y los débiles.

- Definición y justificación de los objetivos y de su prioridad para la atención del problema.

- Identificación y análisis de alternativas de solución, sin excluir ninguna alternativa generada inicialmente.

- Decisión a partir del análisis realizado.

- Plan de acción para conocer el camino a seguir.

Se aprecia en las anteriores herramientas de gestión que los dos puntos principales están relacionados con identificar qué origina la problemática y tener claridad del objetivo para lograr una lluvia de ideas que permita la reflexión crítica en su selección.

\section{Evaluación permanente}

Por último, uno de los aspectos clave es la sensibilización hacia una cultura que inicie desde un sistema de medición del desempeño y que, a su vez, sea parte de una evaluación organizacional. Esto último no solo implica establecer indicadores de medición, sino que, en cada acción, las acciones evaluativas sean vistas como parte de todo un proceso y sean inherentes en el quehacer institucional. En este marco colectivo, el sentir individual de mejorar y de ser autorreflexivo conduce a coadyuvar al fin último de la organización.

La UNED, como universidad pública, está llamada a crear valor público a la sociedad y, al comprender esta finalidad, la responsabilidad y el compromiso como partes de la comunidad universitaria adquieren mayor peso y obligan a adoptar las mejores prácticas en gestión organizacional.

Bajo esta línea, surge el concepto del valor público, como pieza del triángulo estratégico, en el que los resultados, condicionantes e implementación se interrelacionan, para poder trascender de la forma tradicional de ver la gestión pública hacia un nivel de entendimiento mayor.

El triángulo estratégico en la administración pública posiciona al valor público como parte de esta tríada. El valor público definido como "el valor público que crean diferentes actores sociales" (Gutiérrez F (2016), "El Valor Público en Países Democráticos en Costa Rica” (material de curso), Alajuela, Costa Rica”

El valor público en países democráticos se caracteriza por gran cantidad de necesidades por atender, con recursos limitados, que implica una movilización de recursos de la comunidad, apoyo de actores externos, existencia de medios de comunicación para informar y educar a la ciudadanía, y un proceso educativo cívico para que todos los participantes comprendan que implica un cambio adaptativo profundo.

Las dimensiones del valor público en instituciones públicas en países en desarrollo contemplan el bienestar humano, entendido como aquello que permite que las personas puedan tener vidas significativas y valiosas, mediante el desarrollo ético y social, al permitir la equidad, dignidad e integración social; la economía productiva, que se refiere a la existencia de oportunidades para el crecimiento, la estabilidad y la solvencia económica; la sostenibilidad ambiental, relacionada con el desarrollo ambiental y la protección de los recursos del planeta; y la gobernabilidad y la participación, relacionadas con personas organizadas, comprometidas, educadas y capaces de fortalecer la democracia, la seguridad y la convivencia. Las entidades tienen incidencia en la gobernabilidad y la participación en la medida en que, como tomadoras de decisiones, influyen en las otras dimensiones.

De todo lo anterior, la propuesta concreta de esta sistematización de experiencia se fundamenta en que la mejora de una gestión pública inicia desde el quehacer individual, en que el conocimiento es poder, y debe ser visto como herramienta 
indispensable para comprender no solo el ambiente inmediato, sino el entorno y el contexto organizacional, nacional e internacional. Aunado a lo anterior, no puede dejarse de lado desarrollar habilidades en liderazgo emocional y toma de decisiones; $y$, junto a esto, un sistema permanente de desempeño y acciones evaluativas con criticidad y que funcionen como un proceso autorregulado que incida en un macro organizacional, el cual contribuya a lograr el fin último que genera valor público a la sociedad.

\section{METODOLOGÍA}

La construcción de la sistematización de la experiencia presentada en estas páginas responde al paradigma interpretativo, cuyo interés es "comprender, interpretar y compartir la comprensión de forma mutua y participativa" (Koetthing, citado por Ricoy, 2006, pág. 14), aplicando como técnica la perspectiva de la persona participante, basándose en una investigación con enfoque cualitativo.

En este sentido, cabe resaltar que una de las clasificaciones de la sistematización de experiencias, según el sujeto, es aquella centrada en el proceso de intervención participativa, definida como la "producción de conocimiento basada en la comunicación y recuperación de experiencias vividas, con la participación de todos los actores sociales” (Grupo Alforja Centroamérica, citado por Pérez de Maza, 2016, pág.11).

La sistematización de experiencias, según menciona Jara (citado por Pérez de Maza, 2016, pág. 13), es una “interpretación crítica de una o varias experiencias que, a partir de su ordenamiento y reconstrucción, descubre o explicita la lógica del proceso, los factores que han intervenido en él, cómo se han relacionado entre sí y por qué lo han hecho de ese modo”.

La sistematización es un proceso complejo de análisis, reflexión, comunicación y aprendizaje. Como lo señalan Tapella y Rodriguez-Billela (2014, pág.86), “(...) la sistematización de experiencias también puede ser comprendida como parte del amplio movimiento de la gestión del conocimiento, integrándose a la par de enfoques como la capitalización de experiencias y la documentación de procesos".

Como indica Sánchez (2016, la sistematización presenta cuatro características: es una actividad reflexiva, se aplica a un proceso concreto, permite organizar un conjunto de elementos que pueden estar desarticulados y es un proceso que involucra a las personas relacionadas con la experiencia.

Acevedo (citado por Sánchez, 2016) menciona que, metodológicamente, la sistematización se puede realizar en tres fases, no necesariamente secuenciales:

1. La reconstrucción: se relaciona con la descripción de la experiencia y la interpretación que hacen de ella las personas involucradas.

2. La interpretación: permite identificar temas recurrentes y significados que los actores le dan a la experiencia.

3. La potenciación de la experiencia: es una comprensión de la experiencia desde su perspectiva transformadora y sus posibilidades futuras (Acevedo, 2008, citado por Sánchez (2016), pp.24-31).

En este sentido, como parte de las técnicas de recolección de datos en la sistematización de experiencias, se menciona el uso del benchmarking, entendida como

una herramienta fundamental en la búsqueda externa de ideas, estrategias y métodos para el perfeccionamiento de la propia organización. Se trata del proceso sistemático y continuado para evaluar y comparar los productos, los servicios y los procesos de trabajo de las organizaciones que se reconocen como representantes de las mejores prácticas, con el propósito de realizar procesos organizacionales. (Spendolini, citado por Marciniak, 2017, pág. 6). 
Esta herramienta permitió valorar comparativamente los principales elementos de éxito en el ámbito público, por medio del intercambio de experiencias y opiniones a nivel de otras organizaciones a nivel latinoamericano, bajo la premisa de compartir información mutua en un marco teórico-práctico de aprendizaje que permitiera analizar las diferencias existentes y replicar los factores que hacen distinción para el reconocimiento de un mejor desempeño en la gestión pública.

\section{RESULTADOS: Reflexión para la gestión de la UNED}

A partir lo anterior, se establece una pregunta fundamental: ¿cómo se puede mejorar a la gestión dentro de la UNED a partir de estos elementos?

Como lo demuestra el índice de competitividad global, la educación superior es un factor que potencia la eficiencia de un país y en el cual Costa Rica obtiene una calificación destacada, pero con elementos a mejorar.

Entre ellos, la calidad de enseñanza en el área de matemáticas y ciencias básicas y el nivel o alcance de capacitación en formación del talento humano que realizan las organizaciones (Foro Económico Mundial, 2017).

A partir de esta información, el índice resulta ser una fuente de información global de gran importancia, tanto para la toma de decisiones como para la generación de acciones y política universitaria, con miras a contrarrestar las deficiencias mostradas en el informe. Sumado a esto, identificar los mejores países a nivel global en las diferentes áreas permitirá mejorar el desempeño del país, si se realiza un análisis comparativo de las acciones que realizan. Por ejemplo, para el caso que atañe a la educación superior, el modelo de Finlandia es un desafío a estudiar y replicar sus buenas prácticas.

Como complemento a lo anterior, en el mundo actual de las organizaciones se hace imperativo el conocimiento de indicadores económicos que permitan el entendimiento del entorno en el que está inmersa la universidad. Ejemplo de ello es el comportamiento de la producción medida por el producto interno bruto (PIB) y el nivel de empleo; las cuentas del sector externo, como lo es la balanza de pagos, la deuda externa y las reservas monetarias internacionales; las finanzas del sector público y el nivel de inflación, tasa de interés y salarios.

En el caso de las universidades públicas, es fundamental este marco de acción ante los escenarios de negociación del Fondo Especial para la Educación Superior Universitaria (FEES), considerando el contexto y el planteamiento de acciones universitarias tendientes a generar conocimiento, diálogo y acciones para fortalecer y comprender las variables económicas.

Con relación al índice de progreso social, es relevante el factor de educación superior como elemento clave en el eje de oportunidad. Cobra relevancia para la universidad analizar con detenimiento la política universitaria y su vinculación con el Gobierno, empresas y organizaciones de la sociedad civil que consideren este índice como marco de acción futuro, convirtiéndose así en una herramienta para la toma de decisiones de las inversiones y acciones a definir. El IDS se transforma en una manera de medir el seguimiento de los objetivos de desarrollo sostenible (ODS), al servir como complemento a los sistemas de seguimiento y evaluación que se definan a nivel organizacional para el monitoreo del cumplimiento de los objetivos de desarrollo.

Dicho lo anterior, surge otra interrogante de análisis: desde el accionar individual, ¿cómo se puede mejorar la gestión universitaria?

Como una posible respuesta, es importante traer a colación que la administración, en su concepto básico, se define como el "proceso de trabajar con las personas y con los recursos para lograr las metas de la organización” (Bateman y Snell, 1999, pág. 6). Derivado de este concepto, una de las funciones tradicionales es la dirección del líder para estimular un desempeño elevado por parte de las personas colaboradoras. Es por ello que resulta fundamental la gestión del talento humano por medio de una visión con liderazgo indistintamente del tipo de organización del que se trate. 
Como indica Peter Drucker,

cada vez será más frecuente que la capacidad de las organizaciones -y no solo de las empresas-para sobrevivir dependa de su ventaja competitiva para lograr que los trabajadores del conocimiento sean más productivos. La capacidad de atraer y de retener a los mejores trabajadores del conocimiento es el primer y más importante requisito previo (citado por Jericó, 2008, pág. 129).

En este sentido, cobra relevancia el liderazgo emocional que se puede desarrollar en todos los niveles de la organización. Como bien lo indican Goleman, Boyatzis y McKee (2013, xviii), "hay muchos líderes, no solamente uno. Reside no solamente en la cima, sino en cada persona y nivel, de un modo u otro".

De ahí que el modelo de cambio intencionado se convierta en una herramienta de gran valía para la transformación personal y liderazgo, en la que se destaca la importancia de conocer en qué escenario se está y hacia dónde se quiere ir en el futuro, permitiendo con ello compartir una visión en conjunto.

Otra de las habilidades directivas que se destacan es la toma de decisiones efectiva y, para ello, conocer y apropiarse del modelo de Harvard de toma de decisiones se suma al conocimiento teórico y práctico para mejorar el desempeño. En el análisis de los problemas decisionales, la práctica lleva a la excelencia, por lo que cada vez se debe comprender el entorno institucional, trabajar en equipo y rechazar el statu quo.

Dicho lo anterior, la mejora en el análisis y la reflexión individual pasan a través del desarrollo de habilidades blandas o de una inteligencia emocional aplicada al liderazgo. Ello permite mejorar el clima organizacional y el desempeño profesional, lo que coadyuva a una gestión eficiente dentro de la institución.

Como reflexión final, la gestión universitaria debe contar con una fuerte cultura de rendición de cuentas y evaluación, con el fin de poder responder al valor público que la universidad, como casa de enseñanza, le debe a la sociedad. Aquellas personas que formamos parte debemos adquirir los conocimientos pertinentes para desarrollar una actitud crítica y fortalecer las habilidades necesarias para elevar por medio de un proceso constante el bienestar colectivo.

\section{CONCLUSIONES}

Ha quedado de manifiesto que la competitividad de un país está determinada por el índice de competitividad global y, recientemente, por el índice de progreso social. En ambos se destaca la importancia de la educación y, para el caso que nos atañe, del sistema de educación superior universitario. El uso y comprensión de estos índices permite analizar las brechas que se generan a nivel mundial y regional, y así comparar los puntos de encuentro y desencuentro. De esta forma, puede visualizarse que estudiar estos temas puede convertirse en una útil herramienta de toma de decisiones para encauzar los esfuerzos de la UNED hacia el logro de su misión y visión, enfocadas en la generación del valor público.

Hoy, más que nunca, estamos en una coyuntura donde la universidad pública debe generar una cultura evaluativa y de rendición de cuentas con mayor fuerza ante la sociedad costarricense y, en el caso de la UNED, aportar en forma categórica a una mayor cobertura, equidad y acceso en las diferentes regiones del país, aspectos clave para generar oportunidades de educación que inciden en la competitividad nacional.

El presente trabajo es un aporte a la reflexión de la gestión pública, en la que se propone el análisis de la oportunidad de visionar la creación de equipos de alto desempeño, con la claridad de que se requiere desarrollar habilidades blandas y crear condiciones adecuadas a nivel institucional para sensibilizar, promover e implementar acciones afirmativas en la generación de una cultura enfocada en el talento humano. 
Otro de los aportes en esta línea es la toma de decisiones y cómo puede mejorarse en el quehacer diario. En párrafos anteriores se consideró el modelo de Harvard, el cual, aunque parezca sencillo, requiere un análisis previo y estructurado para incrementar este proceso. Evidentemente, la teoría debe llevarse a la práctica y, conforme se presenten diferentes momentos decisorios, la particularidad y el contexto deben tomarse en cuenta en la aplicación.

Finalmente, la evaluación y la rendición de cuentas son factores que deben permear la gestión universitaria, no solo para informar a la sociedad con transparencia y responsabilidad, sino también por la generación del valor público de la universidad como institución de educación superior.

Este aporte inicial surge de la teoría de una experiencia académica con países de Latinoamérica como Panamá, Honduras, Perú, Venezuela, Paraguay y Costa Rica, en conjunto con una vivencia práctica del empréstito con el Gobierno, las universidades públicas y el Banco Mundial, donde la UNED tuvo la oportunidad de participar como ejecutora del Proyecto de Mejoramiento de la Educación Superior. En este sentido, la evidencia empírica nos lleva a reflexionar en que el trabajo de cada individuo debe ser pensado como parte de un proceso sistémico, por lo que cada una de sus partes suma un todo, y es en este punto donde radica la importancia de incrementar nuestro desempeño como personas del ámbito público. Es claro que se necesita mayor análisis y reflexión para su comprensión y aplicabilidad, siendo imperante que se genere ese espacio de creación de conocimiento y de discusión académica ligada a experimentar las vivencias que permitirán mejorar la gestión pública. 


\section{REFERENCIAS}

Bateman, T. y Snell, S. (1999). Administración, una ventaja competitiva. México, Editorial McGraw-Hill.

Boyatzis, Richard y McKee, A. (2003). Liderazgo emocional. Estados Unidos: Debolsillo.

Foro Económico Mundial (2016). Índice de Competitividad Global 2016-2017. Suiza.

Foro Económico Mundial (2017). Índice de Competitividad Global 2017-2018. Suiza.

INCAE Business School (2017), Centro Lationamericano para la Competitividad y Desarrollo Sostenible (CLACDS), Costa Rica.

Goleman, D.; Boyatzis, R. y McKee, A. (2013). Primal Leadership. Estados Unidos: Harvard Business Review Press.

Jericó, P. (2008). La nueva Gestión del Talento: construyendo compromiso. Madrid: Prentice Hall.

Marciniak, R. (2017). El benchmarking como herramienta de mejora de la calidad de la educación universitaria virtual. Ejemplo de una experiencia polaca. Educar, 53(1).

Pérez de Maza, T. (2016). Sistematización de experiencias en contextos universitarios. Guía didáctica. Caracas: Universidad Nacional Abierta, Ediciones del Vicerrectorado Académico.

Porter, M. y Stern, S. (2017). Índice de Progreso Social 2017. Estados Unidos.

Sánchez, C. (2016). Una propuesta epistemológica para la sistematización de experiencias generada desde la reflexión sobre la práctica. Revista Interamericana de Educación, Pedagogía y Estudios Culturales, 9(2), julio-diciembre.

Sánchez, A. (2010). El artículo sistematización de experiencias: construcción de sentido desde una perspectiva crítica. Revista Virtual de la Universidad Católica del Norte, 29.

Tapella, E. y Rodriguez-Billela, P. (2014). Sistematización de experiencias: una metodología para evaluar intervenciones de desarrollo. Revista de Evaluación de Programas y Políticas Públicas, 3, 80-116.

Ricoy, C.(2006). Contribución sobre los paradigmas de investigación. Educação, 31(1), undefined-undefined. [fecha de Consulta 29 de Noviembre de 2019]. ISSN: 0101-9031. Disponible en: https://www.redalyc.org/articulo. oa? $\operatorname{id}=1171 / 117117257002$

Universidad Estatal a Distancia. Lineamientos de Política Institucional (2015-2019). 